\title{
VIABILITY RESULT FOR HIGHER-ORDER FUNCTIONAL DIFFERENTIAL INCLUSIONS
}

\author{
MYELKEBIR AITALIOUBRAHIM
}

\begin{abstract}
We prove, in separable Banach spaces, the existence of viable solutions for the following higher-order functional differential inclusion

$$
x^{(k)}(t) \in F\left(t, T(t) x, x^{(1)}(t), \ldots, x^{(k-1)}(t)\right), \quad \text { a.e. on }[0, \tau] .
$$

We consider the case when the right-hand side is nonconvex and the constraint is moving.
\end{abstract}

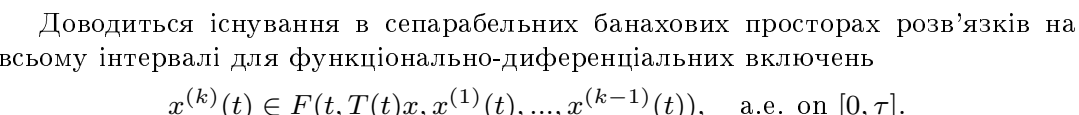

Розглядається випадок неопуклої правої частини та рухомого обмеження.

\section{INTRODUCTION}

Let $E$ be a separable Banach space with a norm $\|\cdot\|$. For $I$ a segment in $\mathbb{R}$, we denote by $\mathcal{C}(I, E)$ the Banach space of continuous functions from $I$ to $E$ equipped with the norm $\|x(\cdot)\|_{\infty}:=\sup \{\|x(t)\| ; t \in I\}$ and $W^{n, 1}(I, E)$ the space of functions possessing absolutely continuous derivatives up to order $n-1$. For $a$ a positive number, we put $\mathcal{C}_{a}:=\mathcal{C}([-a, 0], E)$ and for any $t \in[0, \tau], \tau>0$, we define the operator $T(t)$ from $\mathcal{C}([-a, \tau], E)$ to $\mathcal{C}_{a}$ with $(T(t)(x())).(s):=(T(t) x)(s):=x(t+s), \forall s \in[-a, 0]$.

In this paper, we shall prove the existence of solutions to the following functional differential inclusion:

$$
\left\{\begin{array}{l}
x^{(k)}(t) \in F\left(t, T(t) x, x^{(1)}(t), \ldots, x^{(k-1)}(t)\right), \quad \text { a.e. on }[0, \tau], \\
x(s)=\varphi(s), \text { for all } s \in[-a, 0], \\
x^{(i)}(t) \in \Omega_{i}, \text { for all } i=1, \ldots, k-1, \text { and for all } t \in[0, \tau], \\
x(t) \in C(t), \quad \forall t \in[0, \tau],
\end{array}\right.
$$

where $F$ and $C$ are set-valued maps, $\varphi \in \mathcal{C}_{a}, \Omega_{i} \subset E$ for all $i=0, \ldots, k-1$ and $k \geq 1$.

Existence of viability result for functional differential inclusions was first suggested by Haddad [10, 11], when the right-hand side is upper semicontinuous with convex and compact values, in finite dimensional vector space. This program of research on this subject was studied by several authors under various assumptions. In this way we refer the reader to $[2,4,5,9,12]$ and the references therein.

Marco and Morillo, in [13], have established the existence of viable solutions, in finitedimensional vector space, to the following problem

$$
\left\{\begin{array}{l}
x^{(k)}(t) \in F\left(x(t), x^{(1)}(t), \ldots, x^{(k-1)}(t)\right), \quad \text { a.e. on }[0, \tau], \\
x(t) \in K, \quad \forall t \in[0, \tau],
\end{array}\right.
$$

where $F$ is upper semi-continuous set valued-map with compact convex values and $K$ is a set in $E$ such that the graph of $A_{K}^{(k-1)}$, defined below, is locally compact.

2020 Mathematics Subject Classification. 34A60, 49J52.

Keywords. Multifunction, measurability, selection, functional differential inclusion. 
Recently, in [1], Aitalioubrahim and Sajid have established the existence of viable solutions to (1.1), without memory, in Banach space when $C$ is fixed and $F$ is integrably bounded, measurable with respect to the first argument and Lipschitz continuous with respect to the third argument. In addition, they have considered the following tangential condition:

$$
\liminf _{h \rightarrow 0^{+}} \frac{1}{h^{k}} d\left(x+\sum_{i=1}^{k-1} \frac{h^{i}}{i !} y^{i}+\frac{h^{k-1}}{k !} \int_{t}^{t+h} F(s, x) d s, K\right)=0 .
$$

This work extends results which are presented in $[1,2,3,4,5,8,12]$. Indeed, we get an existence result, in a separable Banach space, for higher-order functional differential inclusions, with a constraint which depends on time. The right-hand side verifies the weaker hypotheses. As is known, viability problems need tangential conditions. For the problem (1.1), we shall use a tangency condition which is weaker than that used in [3].

The paper is organized as follows. In Section 2, we recall some preliminary facts that we need in the sequel. In Section 3, we prove the existence of solutions for (1.1).

\section{Preliminaries and statement of the main Result}

For measurability purpose, $E$ (resp. $\Omega \subset E$ ) is endowed with the $\sigma$-algebra $B(E)$ (resp. $B(\Omega)$ ) of Borel subsets for the strong topology and $[0,1]$ is endowed with Lebesgue measure and the $\sigma$-algebra of Lebesgue measurable subsets. For $x \in E$ and $r>0$ let $B(x, r):=\{y \in E ;\|y-x\|<r\}$ be the open ball centered at $x$ with radius $r$ and $\bar{B}(x, r)$ be its closure and put $B=B(0,1)$. For $\varphi(.) \in \mathcal{C}_{a}$ let $B_{a}(\varphi(), r):.=$ $\left\{\psi(.) \in \mathcal{C}_{a} ;\|\varphi(.)-\psi(.)\|_{\infty}<r\right\}$ and let $\bar{B}_{a}(\varphi(), r$.$) be its closure. For x \in E$ and for nonempty subsets $A, B$ of $E$ we denote $d_{A}(x)$ or $d(x, A)$ the real inf $\{\|y-x\| ; y \in A\}$, $e(A, B):=\sup \left\{d_{B}(x) ; x \in A\right\}$ and $H(A, B)=\max \{e(A, B), e(B, A)\}$. A multifunction is said to be measurable if its graph is measurable.

Let us recall the following Lemmas that will be used in the sequel.

Lemma 2.1. [14] Let $G:[a, b] \rightarrow 2^{E}$ be a measurable multifunction and $y():.[a, b] \rightarrow E$ a measurable function. Then for any positive measurable function $r():.[a, b] \rightarrow \mathbb{R}^{+}$, there exists a measurable selection $g($.$) of G$ such that for almost all $t \in[a, b]$

$$
\|g(t)-y(t)\| \leq d(y(t), G(t))+r(t) .
$$

Lemma 2.2. [6]Let $\preceq$ be a given preorder on the nonempty set $\mathcal{B}$ and let $\phi: \mathcal{B} \rightarrow$ $\mathbb{R} \cup\{+\infty\}$ be an increasing function. Suppose that each increasing sequence in $\mathcal{B}$ is majorated in $\mathcal{B}$. Then, for each $x_{0} \in \mathcal{B}$, there exists $x_{1} \in \mathcal{B}$ such that $x_{0} \preceq x_{1}$ and $\phi\left(x_{1}\right)=\phi(x)$ if $x_{1} \preceq x$.

The above function $\phi$, in [6], is supposed to be finite and bounded from above, but this restriction can be removed by replacing $\phi$ by the function $x \mapsto \arctan \phi(x)$.

Before stating our main result, for any integer $n \geq 1$, we recall the tangent set of $n$-th order denoted by $A_{K}^{n}\left(x_{0}, x_{1}, \ldots, x_{n-1}\right)$ introduced by Marco and Murillo [13] as follows:

$$
A_{K}^{n}\left(x_{0}, x_{1}, \ldots, x_{n-1}\right)=\left\{y \in E: \liminf _{h \rightarrow 0^{+}} \frac{n !}{h^{n}} d\left(\sum_{i=0}^{n-1} \frac{h^{i}}{i !} x_{i}+\frac{h^{n}}{n !} y, K\right)=0\right\} .
$$

For any set-valued map $F$, we denote $G r(F)$ its graph.

We shall use the following hypotheses throughout this paper. 
(H1) $C:[0,1] \rightarrow 2^{E}$ is a set-valued map with closed graph, for $i=1, \ldots, k-1, \Omega_{i}$ is a nonempty open subset in $E$, such that for all $t \in[0,1]$,

$$
C(t) \times \prod_{i=1}^{k-1} \Omega_{i} \subset G r\left(A_{C(t)}^{n}\right)
$$

and $\mathcal{K}:[0,1] \rightarrow \mathcal{C}_{a}$ is a set-valued map defined by

$$
\mathcal{K}(t)=\left\{\varphi \in \mathcal{C}_{a}: \varphi(0) \in C(t)\right\},
$$

(H2) $F: G r(\mathcal{K}) \times \prod_{i=1}^{k-1} \Omega_{i} \rightarrow 2^{E}$ is a set-valued map with nonempty closed values satisfying

(i) $t \mapsto F\left(t, \psi, x_{1}, \ldots, x_{k-1}\right)$ is measurable,

(ii) There exists a function $m(.) \in L^{1}\left([0,1], \mathbb{R}^{+}\right)$such that for all $t \in[0,1]$, $\psi, \phi \in \mathcal{K}(t)$ and $\left(x_{1}, \ldots, x_{k-1}\right),\left(y_{1}, . ., y_{k-1}\right) \in \prod_{i=1}^{k-1} \Omega_{i}$

$H\left(F\left(t, \psi, x_{1}, \ldots, x_{k-1}\right), F\left(t, \phi, y_{1}, \ldots, y_{k-1}\right)\right) \leq m(t) \max \left\{\|\psi-\phi\|_{\infty}, \max _{1 \leq i \leq k-1}\left\|x_{i}-y_{i}\right\|\right\}$,

(iii) There exist $g(),. p(),. q_{1}(),. \ldots, q_{k-1}(.) \in L^{1}\left([0,1], \mathbb{R}^{+}\right)$such that for all $t \in$ $[0,1], \psi \in \mathcal{K}(t)$ and $\left(x_{1}, . ., x_{k-1}\right) \in \prod_{i=1}^{k-1} \Omega_{i}$

$$
F\left(t, \psi, x_{1}, \ldots, x_{k-1}\right) \cap \bar{B}\left(0, g(t)+p(t)\|\psi\|_{\infty}+\sum_{i=1}^{k-1} q_{i}(t)\left\|x_{i}\right\|\right) \neq \emptyset,
$$

(H3) (Tangential condition)For all measurable function $v():.[0,1] \rightarrow E$, for all $\rho>$ $0, t \in[0,1], \psi \in \mathcal{K}(t)$ and $\left(x_{1}, . ., x_{k-1}\right) \in \prod_{i=1}^{k-1} \Omega_{i}$ there exists $f \in S_{v, \rho}\left(\psi, x_{1}, . ., x_{k-1}\right)$ such that

$\liminf _{h \mapsto 0^{+}} \frac{k !}{h^{k}} d\left(\psi(0)+\sum_{i=1}^{k-1} \frac{h^{i}}{i !} x_{i}+\int_{t}^{t+h} \frac{(t+h-s)^{k-1}}{(k-1) !} f(s) d s, C(t+h)\right)=0$

where $S_{v, \rho}\left(\psi, x_{1}, . ., x_{k-1}\right)$ is the set of all $f \in L^{1}([0,1], E)$ such that

$$
f(s) \in F\left(s, \psi, x_{1}, \ldots, x_{k-1}\right) \cap \bar{B}\left(0, g(s)+p(s)\|\psi\|_{\infty}+\sum_{i=1}^{k-1} q_{i}(s)\left\|x_{i}\right\|\right)
$$

and

$$
\|f(s)-v(s)\| \leq d\left(v(s), F\left(s, \psi, x_{1}, \ldots, x_{k-1}\right)\right)+\rho \text { for all } s \in[0,1] .
$$

Remark 2.3. We should point out that, if $F$ satisfies the condition (H2), the set $S_{v, \rho}\left(\psi, x_{1}, \ldots, x_{k-1}\right)$ is nonempty. Indeed, by Theorem III.40 and Theorem III.41 in $[7]$

$$
t \mapsto F\left(t, \psi, x_{1}, \ldots, x_{k-1}\right) \cap \bar{B}\left(0, g(t)+p(t)\|\psi\|_{\infty}+\sum_{i=1}^{k-1} q_{i}(t)\left\|x_{i}\right\|\right)
$$

is measurable. Hence, by Lemma 2.1, there exists $f \in S_{v, \rho}\left(\psi, x_{1}, . ., x_{k-1}\right)$.

In the next section, we shall prove the following result. 
Theorem 2.4. If assumptions (H1)-(H3) are satisfied, then for all $\varphi \in \mathcal{K}(0)$ and $\left(x_{1}, . ., x_{k-1}\right) \in \prod_{i=1}^{k-1} \Omega_{i}$, there exist $\tau>0$ and a map $x(.) \in \mathcal{C}([-a, \tau], E) \cap W^{n, 1}([0, \tau], E)$ such that $x($.$) is a solution of (1.1).$

In all the paper, fix $\varphi \in \mathcal{K}(0)$ and $\left(x_{1}, \ldots, x_{k-1}\right) \in \prod_{i=1}^{k-1} \Omega_{i}$. There exists $r>0$ such that $\Omega_{0}^{i}:=\bar{B}\left(x_{i}, \frac{r}{2}\right) \subset \Omega_{i}$ for all $i=1, \ldots, k-1$. Set also $x_{0}=\varphi(0)$ and $\Omega_{0}^{0}:=\bar{B}\left(x_{0}, \frac{r}{2}\right)$. For $\varepsilon>0$ put

$$
\left.\eta(\varepsilon):=\sup \{\rho \in] 0, \varepsilon]:\left\|\varphi\left(t_{1}\right)-\varphi\left(t_{2}\right)\right\|<\varepsilon \text { if }\left|t_{1}-t_{2}\right| \leq \rho\right\} .
$$

\section{Proof of the main Result}

Let $\tau_{1}, \tau_{2}>0$ be such that

$$
\begin{aligned}
& \int_{0}^{\tau_{2}}\left(\sum_{i=0}^{k-1}\left\|x_{i}\right\|+a+g(t)+p(t)\left(\|\varphi\|_{\infty}+r\right)+\sum_{i=1}^{k-1} q_{i}(t)\left(\left\|x_{i}\right\|+r\right)\right) d t<\frac{r}{2} \\
& \text { and } \int_{0}^{\tau_{1}} m(t) d t<1 .
\end{aligned}
$$

Put

$$
\tau=\inf \left\{\tau_{1}, \tau_{2}, \frac{1}{2} \eta\left(\frac{r}{2}\right), 1\right\} .
$$

For all $0<\varepsilon<a$ and $v(.) \in L^{1}([0,1], E)$, set $\mathcal{B}(\varepsilon, v()$.$) the set of all 4$-tuple $(f, x, \theta, u)_{d}$ where $d \in] 0, \tau], f(),. u(.) \in L^{1}([0, d], E), x():.[-a, d] \rightarrow E$ is a continuous mapping and $\theta():.[0, d] \rightarrow[0, d]$ is a step function such that

(i) $x(t)=\sum_{i=0}^{k-1} \frac{t^{i}}{i !} x_{i}+\int_{0}^{t} \frac{(t-s)^{k-1}}{(k-1) !}(u(s)+f(s)) d s$ for all $t \in[0, d]$;

(ii) $f(t) \in F\left(t, T(\theta(t)) x, x^{(1)}(\theta(t)), \ldots, x^{(k-1)}(\theta(t))\right), u(t) \in \varepsilon B, 0 \leq t-\theta(t) \leq \frac{1}{4} \eta\left(\frac{\varepsilon}{4}\right)$, $x(\theta(t)) \in C(\theta(t))$ and $T(\theta(t)) x \in \bar{B}_{a}(\varphi, r)$ for all $t \in[0, d[$;

(iii) $x(d) \in C(d), T(d) x \in \bar{B}_{a}(\varphi, r)$ and $x^{(i)}(t) \in \Omega_{0}^{i}$ for all $0 \leq i \leq k-1$ and $t \in[0, d]$;

(iv) $\|f(t)-v(t)\| \leq d\left(v(t), F\left(t, T(\theta(t)) x, x^{(1)}(\theta(t)), \ldots, x^{(k-1)}(\theta(t))\right)\right)+\varepsilon$ for all $t \in$ $[0, d[$

(v) $\left\|x^{(k-1)}(t)-x_{k-1}-\int_{0}^{t} f(\tau) d \tau\right\| \leq \varepsilon t$ for all $t \in[0, d]$.

Proposition 3.1. If assumptions (H1)-(H3) are satisfied, then for all $0<\varepsilon<a$, and $v(.) \in L^{1}([0,1], E)$, there exists at least one $(f, x, \theta, u)_{\tau} \in \mathcal{B}(\varepsilon, v()$.$) .$

Proof. Let $0<\varepsilon<a$ and $v(.) \in L^{1}([0,1], E)$ be fixed. Put

$$
x(t)=\varphi(t), \forall t \in[-a, 0] .
$$

By the tangential condition, there exist $f_{0} \in S_{v, \varepsilon}\left(\varphi, x_{1}, \ldots, x_{k-1}\right)$ and $\left.\left.h_{0} \in\right] 0, \inf \left\{\tau, \frac{1}{4} \eta\left(\frac{\varepsilon}{4}\right)\right\}\right]$, such that

$$
\frac{k !}{h_{0}^{k}} d\left(\sum_{i=0}^{k-1} \frac{h_{0}^{i}}{i !} x_{i}+\int_{0}^{h_{0}} \frac{\left(h_{0}-s\right)^{k-1}}{(k-1) !} f_{0}(s) d s, C\left(h_{0}\right)\right) \leq \frac{\varepsilon}{2} .
$$

Then there exists $y_{0} \in C\left(h_{0}\right)$ such that

$$
\frac{k !}{h_{0}^{k}}\left\|y_{0}-\sum_{i=0}^{k-1} \frac{h_{0}^{i}}{i !} x_{i}-\int_{0}^{h_{0}} \frac{\left(h_{0}-s\right)^{k-1}}{(k-1) !} f_{0}(s) d s\right\| \leq \varepsilon .
$$


Set

$$
u_{0}=\frac{k !}{h_{0}^{k}}\left(y_{0}-\sum_{i=0}^{k-1} \frac{h_{0}^{i}}{i !} x_{i}-\int_{0}^{h_{0}} \frac{\left(h_{0}-s\right)^{k-1}}{(k-1) !} f_{0}(s) d s\right) .
$$

We get

$$
y_{0}=\sum_{i=0}^{k-1} \frac{h_{0}^{i}}{i !} x_{i}+\frac{h_{0}^{k}}{k !} u_{0}+\int_{0}^{h_{0}} \frac{\left(h_{0}-s\right)^{k-1}}{(k-1) !} f_{0}(s) d s .
$$

Set $d_{0}=h_{0}, u_{0}(s)=u_{0}$ and

$$
x_{0}(t)=\sum_{i=0}^{k-1} \frac{t^{i}}{i !} x_{i}+\frac{t^{k}}{k !} u_{0}+\int_{0}^{t} \frac{(t-s)^{k-1}}{(k-1) !} f_{0}(s) d s, \forall t \in\left[0, d_{0}\right] .
$$

Remark that, for all $i=0, \ldots, k-1$

$$
x_{0}^{(i)}(t)=\sum_{j=0}^{k-1-i} \frac{t^{j}}{j !} x_{j+i}+\int_{0}^{t} \frac{(t-s)^{k-1-i}}{(k-1-i) !}\left(u_{0}(s)+f_{0}(s)\right) d s
$$

We deduce that

$$
\begin{aligned}
& \left\|x_{0}^{(i)}(t)-x_{i}\right\| \\
\leq & \sum_{j=1}^{k-1-i} h_{0}\left\|x_{j+i}\right\|+\int_{0}^{h_{0}}\left(a+g(s)+p(s)\|\varphi\|_{\infty}+\sum_{i=1}^{k-1} q_{i}(s)\left\|x_{i}\right\|\right) d s \\
= & \int_{0}^{h_{0}}\left(\sum_{j=1}^{k-1-i}\left\|x_{j+i}\right\|+a+g(s)+p(s)\|\varphi\|_{\infty}+\sum_{i=1}^{k-1} q_{i}(s)\left\|x_{i}\right\|\right) d s \\
\leq & \frac{r}{2}
\end{aligned}
$$

then $x_{0}^{(i)}(t) \in \Omega_{0}^{i}$ for all $i=0, \ldots, k-1$ and for all $t \in\left[0, d_{0}\right]$. Also, for all $t \in\left[0, d_{0}\right]$

$$
\left\|x_{0}^{(k-1)}(t)-x_{k-1}-\int_{0}^{t} f_{0}(s) d s\right\| \leq \varepsilon t .
$$

Now, let $s \in[-a, 0]$. If $s \leq-d_{0}$, we have

$$
\left\|T\left(d_{0}\right) x(s)-\varphi(s)\right\|=\left\|x\left(s+d_{0}\right)-\varphi(s)\right\|=\left\|\varphi\left(s+d_{0}\right)-\varphi(s)\right\| \leq r
$$

because $d_{0} \leq \frac{1}{2} \eta\left(\frac{r}{2}\right)$. If $s \geq-d_{0}$, one has

$$
\begin{aligned}
\left\|T\left(d_{0}\right) x(s)-\varphi(s)\right\| & =\left\|x\left(s+d_{0}\right)-\varphi(s)\right\| \\
& \leq\left\|x\left(s+d_{0}\right)-\varphi(0)\right\|+\|\varphi(s)-\varphi(0)\| \\
& \leq \frac{r}{2}+\frac{r}{2}
\end{aligned}
$$

because $|s| \leq \frac{1}{2} \eta\left(\frac{r}{2}\right)$ and $x\left(s+d_{0}\right) \in \Omega_{0}^{0}$. From the above, we conclude that $T\left(d_{0}\right) x \in$ $\bar{B}_{a}(\varphi, r)$. Next, set $\theta_{0}(t)=0$ for all $t \in\left[0, d_{0}\right]$. It is clair that $\left(f_{0}, x_{0}, \theta_{0}, u_{0}\right)_{d_{0}} \in \mathcal{B}(\varepsilon, v()$.$) .$ Hence $\mathcal{B}(\varepsilon, v().) \neq \emptyset$. Now, consider the following preorder:

$$
\begin{aligned}
& \left(f_{1}, x_{1}, \theta_{1}, u_{1}\right)_{d_{1}} \preceq\left(f_{2}, x_{2}, \theta_{2}, u_{2}\right)_{d_{2}} \\
\Leftrightarrow \quad & d_{1} \leq d_{2}, f_{1}=\left.f_{2}\right|_{\left[0, d_{1}\right]}, x_{1}=\left.x_{2}\right|_{\left[0, d_{1}\right]}, \theta_{1}=\left.\theta_{2}\right|_{\left[0, d_{1}\right]}, u_{1}=\left.u_{2}\right|_{\left[0, d_{1}\right]}
\end{aligned}
$$

and let $\phi: \mathcal{B}(\varepsilon, v().) \rightarrow \mathbb{R}$ be the function defined by $\phi\left((f, x, \theta, u)_{d}\right)=d$ for all $(f, x, \theta, u)_{d} \in \mathcal{B}(\varepsilon, v()$.$) . By definition, \phi$ is increasing on $\mathcal{B}(\varepsilon, v()$.$) . On the other hand, if$ 
$\left(\left(f_{i}, x_{i}, \theta_{i}, u_{i}\right)_{d_{i}}\right)_{i \in \mathbb{N}}$ is an increasing sequence in $\mathcal{B}(\varepsilon, v()$.$) , we construct a majorant of$ $\left(\left(f_{i}, x_{i}, \theta_{i}, u_{i}\right)_{d_{i}}\right)_{i \in \mathbb{N}}$ as follows:

$$
d=\lim _{i} d_{i}, f(t)=f_{i}(t), \theta(t)=\theta_{i}(t), u(t)=u_{i}(t), \forall t \in\left[0, d_{i}\right]
$$

and

$$
x(t)=\sum_{i=0}^{k-1} \frac{t^{i}}{i !} x_{i}+\int_{0}^{t} \frac{(t-s)^{k-1}}{(k-1) !}(u(s)+f(s)) d s, \forall t \in[0, d] .
$$

We claim that $(f, x, \theta, u)_{d} \in \mathcal{B}(\varepsilon, v()$.$) . Indeed, for all i \in \mathbb{N}$, we have $x\left(d_{i}\right)=x_{i}\left(d_{i}\right) \in$ $C\left(d_{i}\right)$. Since the graph of $C$ is closed, we conclude that $x(d) \in C(d)$. By the same argument, we get $x^{(i)}(d) \in \Omega_{0}^{i}$ for all $0 \leq i \leq k-1$. The other assertions are obvious.

Next, for applying Lemma 2.2, we need the following Claim.

Claim 3.2. For all $(f, x, \theta, u)_{d} \in \mathcal{B}(\varepsilon, v()$.$) with d<\tau$, there exists $(\bar{f}, \bar{x}, \bar{\theta}, \bar{u})_{\bar{d}} \in$ $\mathcal{B}(\varepsilon, v()$.$) such that (f, x, \theta, u)_{d} \preceq(\bar{f}, \bar{x}, \bar{\theta}, \bar{u})_{\bar{d}}$ and $\phi\left((f, x, \theta, u)_{d}\right)<\phi\left((\bar{f}, \bar{x}, \bar{\theta}, \bar{u})_{\bar{d}}\right)$.

Proof. Let $(f, x, \theta, u)_{d} \in \mathcal{B}(\varepsilon, v()$.$) with d<\tau$. For $T(d) x \in \mathcal{K}(d)$ and $\left(x^{(1)}(d), \ldots, x^{(k-1)}(d)\right) \in \prod_{i=1}^{k-1} \Omega_{i}$, by the tangential condition, there exist $\tilde{f} \in$ $S_{v, \varepsilon}\left(T(d) x, x^{(1)}(d), \ldots, x^{(k-1)}(d)\right)$ and $\left.\left.h \in\right] 0, \inf \left\{\tau-d, \frac{1}{4} \eta\left(\frac{\varepsilon}{4}\right)\right\}\right]$, such that

$$
\frac{k !}{h^{k}} d\left(\sum_{i=0}^{k-1} \frac{h^{i}}{i !} x^{(i)}(d)+\int_{d}^{d+h} \frac{(d+h-s)^{k-1}}{(k-1) !} \tilde{f}(s) d s, C(d+h)\right) \leq \frac{\varepsilon}{2} .
$$

Then there exists $y_{1} \in C(d+h)$ such that

$$
\frac{k !}{h^{k}}\left\|y_{1}-\sum_{i=0}^{k-1} \frac{h^{i}}{i !} x^{(i)}(d)-\int_{d}^{d+h} \frac{(d+h-s)^{k-1}}{(k-1) !} \tilde{f}(s) d s\right\| \leq \varepsilon .
$$

Set

$$
u_{1}=\frac{k !}{h^{k}}\left(y_{1}-\sum_{i=0}^{k-1} \frac{h^{i}}{i !} x^{(i)}(d)-\int_{d}^{d+h} \frac{(d+h-s)^{k-1}}{(k-1) !} \tilde{f}(s) d s\right) .
$$

We have

$$
y_{1}=\sum_{i=0}^{k-1} \frac{h^{i}}{i !} x^{(i)}(d)+\frac{h^{k}}{k !} u_{1}+\int_{d}^{d+h} \frac{(d+h-s)^{k-1}}{(k-1) !} \tilde{f}(s) d s .
$$

Next, set $\bar{d}=d+h$,

$$
\tilde{x}(t)=\sum_{i=0}^{k-1} \frac{(t-d)^{i}}{i !} x^{(i)}(d)+\frac{(t-d)^{k}}{k !} u_{1}+\int_{d}^{t} \frac{(t-s)^{k-1}}{(k-1) !} \tilde{f}(s) d s
$$

for all $t \in[d, \bar{d}]$. We define $\bar{f}, \bar{x}, \bar{u}$ and $\bar{\theta}$ as follows:

$$
\bar{f}(t)=f(t), \bar{x}(t)=x(t), \bar{\theta}(t)=\theta(t), \bar{u}(t)=u(t), \text { for all } t \in[0, d]
$$

and

$$
\left.\left.\bar{f}(t)=\tilde{f}(t), \bar{x}(t)=\tilde{x}(t), \bar{\theta}(t)=d, \bar{u}(t)=u_{1}, \text { for all } t \in\right] d, \bar{d}\right] .
$$

We can easily show that, for all $t \in[0, \bar{d}]$

$$
\bar{x}(t)=\sum_{i=0}^{k-1} \frac{t^{i}}{i !} x_{i}+\int_{0}^{t} \frac{(t-s)^{k-1}}{(k-1) !}(\bar{u}(s)+\bar{f}(s)) d s
$$


and for $i=0, \ldots, k-1$, one has

$$
\bar{x}^{(i)}(t)=\sum_{j=0}^{k-1-i} \frac{t^{j}}{j !} x_{j+i}+\int_{0}^{t} \frac{(t-s)^{k-1-i}}{(k-1-i) !}(\bar{u}(s)+\bar{f}(s)) d s .
$$

Then for all $t \in[0, \bar{d}]$

$$
\left\|\bar{x}^{(k-1)}(t)-x_{k-1}-\int_{0}^{t} \bar{f}(s) d s\right\| \leq \varepsilon t
$$

and by (3.4)

$$
\begin{aligned}
& \left\|\bar{x}^{(i)}(t)-x_{i}\right\| \\
\leq & \left\|\sum_{j=1}^{k-1-i} \frac{\bar{d}^{j}}{j !} x_{j+i}+\int_{0}^{\bar{d}} \frac{(\bar{d}-s)^{k-1-i}}{(k-1-i) !}(\bar{u}(s)+\bar{f}(s)) d s\right\| \\
\leq & \sum_{j=1}^{k-1-i} \bar{d}\left\|x_{j+i}\right\|+\int_{0}^{\bar{d}}(\|\bar{u}(s)\|+\|\bar{f}(s)\|) d s \\
\leq & \int_{0}^{\bar{d}}\left(\sum_{j=0}^{k-1}\left\|x_{j}\right\|+a+g(s)+p(s)\|T(\bar{\theta}(s)) \bar{x}\|+\sum_{i=1}^{k-1} q_{i}(s)\|\bar{x}(\bar{\theta}(s))\|\right) d s \\
\leq & \int_{0}^{\bar{d}}\left(\sum_{j=0}^{k-1}\left\|x_{j}\right\|+a+g(s)+p(s)\left(\|\varphi\|_{\infty}+r\right)+\sum_{i=1}^{k-1} q_{i}(s)\left(\left\|x_{i}\right\|+r\right)\right) d s \\
\leq & \frac{r}{2}
\end{aligned}
$$

then $\bar{x}^{(i)}(t) \in \Omega_{0}^{i}$ for all $i=0, \ldots, k-1$ and $t \in[0, \bar{d}]$. Now, let $s \in[-a, 0]$. If $s \leq-\bar{d}$, we have

$$
\begin{aligned}
\|T(\bar{d}) \bar{x}(s)-\varphi(s)\| & =\|\bar{x}(s+\bar{d})-\varphi(s)\| \\
& =\|\varphi(s+\bar{d})-\varphi(s)\| \\
& \leq r
\end{aligned}
$$

If $s \geq-\bar{d}$, one has

$$
\begin{aligned}
\|T(\bar{d}) x(s)-\varphi(s)\| & =\|\bar{x}(s+\bar{d})-\varphi(s)\| \\
& \leq\|\bar{x}(s+\bar{d})-\varphi(0)\|+\|\varphi(s)-\varphi(0)\| \\
& \leq r
\end{aligned}
$$

Hence $T(\bar{d}) x \in \bar{B}_{a}(\varphi, r)$. Finally, we conclude that $(\bar{f}, \bar{x}, \bar{\theta}, \bar{u})_{\bar{d}} \in \mathcal{B}(\varepsilon, v()),.(f, x, \theta, u)_{d} \preceq$ $(\bar{f}, \bar{x}, \bar{\theta}, \bar{u})_{\bar{d}}$ and $\phi\left((f, x, \theta, u)_{d}\right)<\phi\left((\bar{f}, \bar{x}, \bar{\theta}, \bar{u})_{\bar{d}}\right)$.

Now, we are ready to complete the proof of Proposition 3.1. From Lemma 2.2, there exists $(f, x, \theta, u)_{d} \in \mathcal{B}(\varepsilon, v()$.$) such that \phi\left((f, x, \theta, u)_{d}\right)=\phi\left((\bar{f}, \bar{x}, \bar{\theta}, \bar{u})_{\bar{d}}\right)$ and $(f, x, \theta, u)_{d} \preceq$ $(\bar{f}, \bar{x}, \bar{\theta}, \bar{u})_{\bar{d}}$ for all $(\bar{f}, \bar{x}, \bar{\theta}, \bar{u})_{\bar{d}} \in \mathcal{B}(\varepsilon, v()$.$) . Moreover, if \phi\left((f, x, \theta, u)_{d}\right)<\tau$, by the last Claim, there exists $(\bar{f}, \bar{x}, \bar{\theta}, \bar{u})_{\bar{d}} \in \mathcal{B}(\varepsilon, v()$.$) such that (f, x, \theta, u)_{d} \preceq(\bar{f}, \bar{x}, \bar{\theta}, \bar{u})_{\bar{d}}$ and $\phi\left((f, x, \theta, u)_{d}\right)<\phi\left((\bar{f}, \bar{x}, \bar{\theta}, \bar{u})_{\bar{d}}\right)$. Hence $\phi\left((f, x, \theta, u)_{d}\right)=\tau$. The proof is complete.

Now we are prepared to prove our Theorem 2.4. Let $\left(\varepsilon_{n}\right)_{n \geq 1}$ be a strictly decreasing sequence of positive scalars such that $0<\varepsilon_{n}<a$ for all $n \geq 1$ and $\sum_{n=1}^{\infty} \varepsilon_{n}<\infty$. In view of Proposition 3.1, we can define inductively sequences $\left(f_{n}(.)\right)_{n \geq 1} \subset L^{1}([0, \tau], E)$, $\left(x_{n}(.)\right)_{n \geq 1} \subset \mathcal{C}([-a, \tau], E)$, and $\left(\theta_{n}(.)\right)_{n \geq 1}, \subset S([0, \tau],[0, \tau])$ where $S([0, \tau],[0, \tau])$ denotes the space of step functions from $[0, \tau]$ into $[0, \tau]$ such that 
(1) $x_{n}(.) \in W^{k, 1}([0, \tau], E), f_{n}(t) \in F\left(t, T\left(\theta_{n}(t)\right) x_{n}, x_{n}^{(1)}\left(\theta_{n}(t)\right), \ldots, x_{n}^{(k-1)}\left(\theta_{n}(t)\right)\right)$, $x_{n}\left(\theta_{n}(t)\right) \in C\left(\theta_{n}(t)\right), T\left(\theta_{n}(t)\right) x_{n} \in \bar{B}_{a}(\varphi, r)$ and $0 \leq t-\theta_{n}(t) \leq \frac{1}{4} \eta\left(\frac{\varepsilon_{n}}{4}\right)$ for all $t \in[0, \tau[$

(2) $x_{n}(\tau) \in C(\tau), T(\tau) x_{n} \in \bar{B}_{a}(\varphi, r)$ and $x_{n}^{(i)}(t) \in \Omega_{0}^{i}$ for $i=0, \ldots, k-1$ and all $t \in\left[0, \tau\left[\right.\right.$ and $x_{n} \equiv \varphi$ on $[-a, 0]$

(3)

$$
\begin{aligned}
& \left\|f_{n+1}(t)-f_{n}(t)\right\| \\
& \quad \leq d\left(f_{n}(t), F\left(t, T\left(\theta_{n+1}(t)\right) x_{n+1}, x_{n+1}^{(1)}\left(\theta_{n+1}(t)\right), \ldots, x_{n+1}^{(k-1)}\left(\theta_{n+1}(t)\right)\right)\right)+\varepsilon_{n+1}
\end{aligned}
$$

for all $t \in[0, \tau[$;

(4) $\left\|x_{n}^{(k-1)}(t)-x_{k-1}-\int_{0}^{t} f_{n}(\tau) d \tau\right\| \leq \varepsilon_{n} t$ for all $t \in[0, \tau]$.

Let us denote the modulus continuity of a function $\psi$ defined on interval $I$ of $\mathbb{R}$ by

$$
\omega(\psi, I, \varepsilon):=\sup \{\|\psi(t)-\psi(s)\| ; s, t \in I,|s-t|<\varepsilon\}, \varepsilon>0 .
$$

In the rest, we need the following claim

Claim 3.3. (a) For all $i=1, \ldots, k$ and $t \in[0, \tau]$

$$
\left\|x_{n}^{(k-i)}(t)\right\| \leq\left\|x_{k-i}\right\|+\ldots+\left\|x_{k-1}\right\|+r+a,
$$

(b) For all $i=0, \ldots, k-1$

$$
\omega\left(x_{n+1}^{(i)}(.),[0, \tau], \frac{1}{2} \eta\left(\frac{\varepsilon_{n}}{4}\right)\right) \leq \varepsilon_{n}\left(\left\|x_{k-i}\right\|+\ldots+\left\|x_{k-1}\right\|+r+a\right),
$$

(c) $\omega\left(x_{n+1}(),.[-a, 0], \frac{1}{2} \eta\left(\frac{\varepsilon_{n}}{4}\right)\right) \leq \frac{\varepsilon_{n}}{4}$,

(d) For all $t \in[0, \tau]$

$$
\begin{array}{r}
\left.\max \left\{\| T\left(\theta_{n}(t)\right) x_{n}-T\left(\theta_{n+1}(t)\right) x_{n+1}\right)\left\|_{\infty}, \max _{1 \leq i \leq k-1}\right\| x_{n+1}^{(i)}\left(\theta_{n+1}(t)\right)-x_{n}^{(i)}\left(\theta_{n}(t)\right) \|_{\infty}\right\} \\
\leq \varepsilon_{n} \delta+\max _{0 \leq i \leq k-1}\left\|x_{n+1}^{(i)}(.)-x_{n}^{(i)}(.)\right\|_{\infty},
\end{array}
$$

where $\delta=1+\left\|x_{1}\right\|+\ldots+\left\|x_{k-1}\right\|+r+a$

Proof. (a) By induction, for $i=1$, by (4) and (3.4), we have for all $t \in[0, \tau]$

$$
\begin{aligned}
\left\|x_{n}^{(k-1)}(t)\right\| \leq & \left\|x_{n}^{(k-1)}(t)-x_{k-1}-\int_{0}^{t} f_{n}(\tau) d \tau\right\|+\left\|x_{k-1}+\int_{0}^{t} f_{n}(\tau) d \tau\right\| \\
\leq & \varepsilon_{n} t+\left\|x_{k-1}\right\|+\int_{0}^{t}\left\|f_{n}(\tau)\right\| d \tau \\
\leq & a+\left\|x_{k-1}\right\| \\
& +\int_{0}^{\tau}\left(g(s)+p(s)\left\|T\left(\theta_{n}(s)\right) x_{n}\right\|_{\infty}+\sum_{i=1}^{k-1} q_{i}(s)\left\|x_{n}^{(i)}\left(\theta_{n}(s)\right)\right\|\right) d s \\
\leq & a+\left\|x_{k-1}\right\| \\
& +\int_{0}^{\tau}\left(g(s)+p(s)\left(\|\varphi\|_{\infty}+r\right)+\sum_{i=1}^{k-1} q_{i}(s)\left(\left\|x_{i}\right\|+r\right)\right) d s \\
\leq & a+\left\|x_{k-1}\right\|+r
\end{aligned}
$$


Then, (a) is satisfied for $i=1$. Let now $l \geq 2$ assume that (a) is satisfied for any $i=1, \ldots, l$. We have for $t \in[0, \tau]$

$$
\begin{aligned}
\left\|x_{n}^{(k-l-1)}(t)-x_{n}^{(k-l-1)}(0)\right\| & \leq \int_{0}^{t}\left\|x_{n}^{(k-l)}(s)\right\| d s \\
& \leq\left\|x_{k-l}\right\|+\ldots+\left\|x_{k-1}\right\|+r+a
\end{aligned}
$$

Then

$$
\left\|x_{n}^{(k-l-1)}(t)\right\| \leq\left\|x_{k-l-1}\right\|+\left\|x_{k-l}\right\|+\ldots+\left\|x_{k-1}\right\|+r+a .
$$

Hence (a) is satisfied for $i=l+1$.

(b) Let $t, t^{\prime} \in[0, \tau]$ with $t<t^{\prime}$. By (a), for $i=0, \ldots, k-1$, we have

$$
\begin{aligned}
\left\|x_{n+1}^{(i)}(t)-x_{n+1}^{(i)}\left(t^{\prime}\right)\right\| & \leq \int_{t}^{t^{\prime}}\left\|x_{n+1}^{(i+1)}(s)\right\| d s \\
& \leq\left(t-t^{\prime}\right)\left(\left\|x_{i+1}\right\|+\ldots+\left\|x_{k-1}\right\|+r+a\right)
\end{aligned}
$$

Then $\omega\left(x_{n+1}^{(i)}(),.[0, \tau], \frac{1}{2} \eta\left(\frac{\varepsilon_{n}}{4}\right)\right) \leq \varepsilon_{n}\left(\left\|x_{i+1}\right\|+\ldots+\left\|x_{k-1}\right\|+r+a\right)$, for all $i=$ $0, \ldots, k-1$.

(c) Let $t, t^{\prime} \in[-a, 0]$ with $t<t^{\prime}$. We have

$$
\left\|x_{n+1}(t)-x_{n+1}\left(t^{\prime}\right)\right\|=\left\|\varphi(t)-\varphi\left(t^{\prime}\right)\right\|
$$

Then by (2.3) one has $\omega\left(x_{n+1}(),.[-a, 0], \frac{1}{2} \eta\left(\frac{\varepsilon_{n}}{4}\right)\right) \leq \frac{\varepsilon_{n}}{4}$.

(d) For $t \in[0, \tau]$, we have

$$
\begin{aligned}
\| T\left(\theta_{n+1}(t)\right) x_{n+1}- & T\left(\theta_{n}(t)\right) x_{n} \|_{\infty} \\
= & \sup _{s \in[-a, 0]}\left\|x_{n+1}\left(\theta_{n+1}(t)+s\right)-x_{n}\left(\theta_{n}(t)+s\right)\right\| \\
\leq & \sup _{s \in[-a, 0]}\left\|x_{n+1}\left(\theta_{n+1}(t)+s\right)-x_{n+1}\left(\theta_{n}(t)+s\right)\right\| \\
& \quad+\sup _{s \in[-a, 0]}\left\|x_{n+1}\left(\theta_{n}(t)+s\right)-x_{n}\left(\theta_{n}(t)+s\right)\right\| \\
\leq & \omega\left(x_{n+1}(.),[-a, \tau], \frac{1}{2} \eta\left(\frac{\varepsilon_{n}}{4}\right)\right)+\sup _{s \in[-a, \tau]}\left\|x_{n+1}(t)-x_{n}(t)\right\| \\
\leq & \omega\left(x_{n+1}(.),[-a, 0], \frac{1}{2} \eta\left(\frac{\varepsilon_{n}}{4}\right)\right)+\omega\left(x_{n+1}(.),[0, \tau], \frac{1}{2} \eta\left(\frac{\varepsilon_{n}}{4}\right)\right) \\
& \quad+\left\|x_{n+1}(.)-x_{n}(.)\right\|_{\infty} \\
\leq & \frac{\varepsilon_{n}}{4}+\varepsilon_{n}\left(\left\|x_{1}\right\|+\ldots+\left\|x_{k-1}\right\|+r+a\right)+\left\|x_{n+1}(.)-x_{n}(.)\right\|_{\infty} \\
\leq & \varepsilon_{n} \delta+\max _{0 \leq i \leq k-1}\left\|x_{n+1}^{(i)}(.)-x_{n}^{(i)}(.)\right\|_{\infty}
\end{aligned}
$$

On the other hand, one has for all $t \in[0, \tau]$ and for all $i=0, \ldots, k-1$,

$$
\begin{aligned}
\| x_{n}^{(i)}\left(\theta_{n}(t)\right) & -x_{n+1}^{(i)}\left(\theta_{n+1}(t)\right) \| \\
& \leq\left\|x_{n}^{(i)}\left(\theta_{n}(t)\right)-x_{n}^{(i)}\left(\theta_{n+1}(t)\right)\right\|+\left\|x_{n}^{(i)}\left(\theta_{n+1}(t)\right)-x_{n+1}^{(i)}\left(\theta_{n+1}(t)\right)\right\| \\
& \leq \omega\left(x_{n}^{(i)}(.),[0, \tau], \frac{1}{2} \eta\left(\frac{\varepsilon_{n}}{4}\right)\right)++\left\|x_{n}^{(i)}\left(\theta_{n+1}(t)\right)-x_{n+1}^{(i)}\left(\theta_{n+1}(t)\right)\right\| \\
& \leq \varepsilon_{n}\left(\left\|x_{i+1}\right\|+\ldots+\left\|x_{k-1}\right\|+r+a\right)+\left\|x_{n}^{(i)}(.)-x_{n+1}^{(i)}(.)\right\|_{\infty} \\
& \leq \varepsilon_{n} \delta+\max _{0 \leq i \leq k-1}\left\|x_{n}^{(i)}(.)-x_{n+1}^{(i)}(.)\right\|_{\infty} .
\end{aligned}
$$


Thus

$\max _{0 \leq i \leq k-1}\left\|x_{n}^{(i)}\left(\theta_{n}(t)\right)-x_{n+1}^{(i)}\left(\theta_{n+1}(t)\right)\right\| \leq \varepsilon_{n} \delta+\max _{0 \leq i \leq k-1}\left\|x_{n}^{(i)}(.)-x_{n+1}^{(i)}(.)\right\|_{\infty}$.

Hence the assertion (d) is proved.

Now, from (1), (3) and the last claim, we deduce for all $t \in[0, \tau[$

$$
\begin{aligned}
\| f_{n+1}(t)- & f_{n}(t) \| \\
\leq & H\left(F\left(t, T\left(\theta_{n}(t)\right) x_{n}, x_{n}^{(1)}\left(\theta_{n}(t)\right), \ldots, x_{n}^{(k-1)}\left(\theta_{n}(t)\right)\right),\right. \\
& \left.F\left(t, T\left(\theta_{n+1}(t)\right) x_{n+1}, x_{n+1}^{(1)}\left(\theta_{n+1}(t)\right), \ldots, x_{n+1}^{(k-1)}\left(\theta_{n+1}(t)\right)\right)\right)+\varepsilon_{n+1} \\
\leq & m(t) \max \left\{\| T\left(\theta_{n}(t)\right) x_{n}-T\left(\theta_{n+1}(t)\right) x_{n+1}\right) \|_{\infty}, \\
& \left.\max _{1 \leq i \leq k-1}\left\|x_{n+1}^{(i)}\left(\theta_{n+1}(t)\right)-x_{n}^{(i)}\left(\theta_{n}(t)\right)\right\|_{\infty}\right\}+\varepsilon_{n+1} \\
\leq & m(t) \varepsilon_{n} \delta+m(t) \max _{0 \leq i \leq k-1}\left\|x_{n+1}^{(i)}(.)-x_{n}^{(i)}(.)\right\|_{\infty}+\varepsilon_{n+1}
\end{aligned}
$$

On the other hand, we have for $i=0, \ldots, k-1$,

$$
x_{n}^{(i)}(t)=\sum_{j=i}^{k-1} \frac{t^{j-i}}{(j-i) !} x_{j}+\int_{0}^{t} \frac{(t-s)^{k-1-i}}{(k-1-i) !}\left(u_{n}(s)+f_{n}(s)\right) d s .
$$

Then

$$
\begin{aligned}
\| x_{n+1}^{(i)}(t)- & x_{n}^{(i)}(t) \| \\
\leq & \int_{0}^{t} \frac{(t-s)^{k-1-i}}{(k-1-i) !}\left(\left\|u_{n+1}(s)-u_{n}(s)\right\|+\left\|f_{n+1}(s)-f_{n}(s)\right\|\right) d s \\
\leq & 2 \varepsilon_{n}+\int_{0}^{t}\left\|f_{n+1}(s)-f_{n}(s)\right\| d s \\
\leq & 2 \varepsilon_{n}+\varepsilon_{n} \delta \int_{0}^{t} m(s) d s \\
& \quad+\max _{0 \leq i \leq k-1}\left\|x_{n+1}^{(i)}(.)-x_{n}^{(i)}(.)\right\|_{\infty} \int_{0}^{t} m(s) d s+\varepsilon_{n+1} \int_{0}^{t} m(s) d s \\
\leq & \varepsilon_{n}(3+\delta)+\max _{0 \leq i \leq k-1}\left\|x_{n+1}^{(i)}(.)-x_{n}^{(i)}(.)\right\|_{\infty} \int_{0}^{1} m(s) d s
\end{aligned}
$$

Thus

$$
\max _{0 \leq i \leq k-1}\left\|x_{n+1}^{(i)}(.)-x_{n}^{(i)}(.)\right\|_{\infty} \leq \frac{\varepsilon_{n}(3+\delta)}{1-L}
$$

where $L=\int_{0}^{1} m(s) d s$. Therefore we have for all $i=0, \ldots, k-1$, and for $n<m$

$$
\left\|x_{n}^{(i)}(.)-x_{m}^{(i)}(.)\right\|_{\infty} \leq \frac{3+\delta}{1-L} \sum_{j=n}^{m-1} \varepsilon_{j} .
$$

So the sequence $\left\{x_{n}^{(i)}(.)\right\}_{n=1}^{\infty}$ for $i=0, \ldots, k-1$, is a Cauchy sequence, then it converges uniformly on $[0, \tau]$ to a function $z_{i}($.$) . Since all functions x_{n}($.$) agree with \varphi$ on $[-a, 0]$, 
we can obviously say that $x_{n}($.$) converges uniformly to z_{0}($.$) on [-a, \tau]$, if we extend $z_{0}($. in such a way that $z_{0}(.) \equiv \varphi$ on $[-a, 0]$. Also, by (c) and the following inequality

$$
\left\|x_{n}\left(\theta_{n}(t)\right)-z_{0}(t)\right\| \leq\left\|x_{n}\left(\theta_{n}(t)\right)-x_{n}(t)\right\|+\left\|x_{n}(t)-z_{0}(t)\right\|,
$$

we deduce that $x_{n}\left(\theta_{n}().\right)$ converges uniformly to $z_{0}($.$) on [0, \tau]$. By construction, we have $x_{n}^{(i)}(t) \in \Omega_{i}^{0}$ for every $t \in[0, \tau]$, then $z_{i}(t) \in \Omega_{i}^{0}$ for all $t \in[0, \tau]$ and $i=0, \ldots, k-1$. In addition, since $x_{n}\left(\theta_{n}(t)\right) \in C\left(\theta_{n}(t)\right)$ for every $t \in[0, \tau]$ and since the graph of $C$ is closed, we get $x(t) \in C(t)$ for all $t \in[0, \tau]$.

Now, we return to the relation (3.5). By the relation (3.6) we get

$$
\begin{aligned}
& \left\|f_{n+1}(t)-f_{n}(t)\right\| \\
\leq & \varepsilon_{n}\left(m(t)\left(\delta+\frac{3+\delta}{1-L}\right)+1\right) .
\end{aligned}
$$

This implies (as above) that $\left\{f_{n}(t)\right\}_{n=1}^{\infty}$ is a Cauchy sequence and $\left(f_{n}(t)\right)_{n}$ converges to $f(t)$. Further, since

$$
\left\|f_{n}(t)\right\| \leq g(t)+p(t)\left(\|\varphi\|_{\infty}+r\right)+\sum_{i=1}^{k-1} q_{i}(t)\left(\left\|x_{i}\right\|+r\right),
$$

by (4) and by the dominated convergence theorem

$$
z_{k-1}(t)=\lim _{n \rightarrow \infty} x_{n}^{(k-1)}(t)=\lim _{n \rightarrow \infty}\left(x_{k-1}+\int_{0}^{t} f_{n}(s) d s\right)=x_{k-1}+\int_{0}^{t} f(s) d s .
$$

Hence $\dot{z}_{k-1}(t)=f(t)$. Also the relation

$$
x_{n}^{(i)}(t)=x_{i}+\int_{0}^{t} x_{n}^{(i+1)}(s) d s, \text { for } i=0, \ldots, k-1
$$

yields

$$
z_{i}(t)=x_{i}+\int_{0}^{t} z_{i+1}(s) d s \text { for } i=0, \ldots, k-1 .
$$

Thus $\dot{z}_{i}(t)=z_{i+1}(t)$ for all $i=0, \ldots, k-1$ and $t \in[0, \tau]$. In addition, by (2.3) and (b), we have

$$
\begin{aligned}
\left\|T\left(\theta_{n}(t)\right) x_{n}-T(t) x_{n}\right\|_{\infty} & =\sup _{-a \leq s \leq 0}\left\|x_{n}\left(\theta_{n}(t)+s\right)-x_{n}(t+s)\right\| \\
& \leq \omega\left(x_{n},[-a, \tau], \frac{1}{2} \eta\left(\frac{\varepsilon_{n}}{4}\right)\right) \\
& \leq \omega\left(\varphi,[-a, 0], \frac{1}{2} \eta\left(\frac{\varepsilon_{n}}{4}\right)\right)+\omega\left(x_{n},[0, \tau], \frac{1}{2} \eta\left(\frac{\varepsilon_{n}}{4}\right)\right) \\
& \leq \frac{\varepsilon_{n}}{4}+\varepsilon_{n}\left(\left\|x_{1}\right\|+\ldots+\left\|x_{k-1}\right\|+r+a\right) \\
& \leq \varepsilon_{n} \delta
\end{aligned}
$$

Hence $\left\|T\left(\theta_{n}(t)\right) x_{n}-T(t) x_{n}\right\|_{\infty}$ converges to 0 as $n \rightarrow+\infty$. Therefore, since the uniform convergence of $x_{n}($.$) to z_{0}($.$) on [-a, \tau]$ implies that $T(t) x_{n}$ converges to $T(t) z_{0}$ uniformly on $[-a, 0]$, we deduce that

$$
T\left(\theta_{n}(t)\right) x_{n} \text { converges to } T(t) z_{0} \text { in } \mathcal{C}_{a} .
$$


Now, observe that by (1),

$$
\begin{aligned}
& d\left(f(t), F\left(t, T(t) z_{0}, z_{1}(t), \ldots, z_{k-1}(t)\right)\right) \\
& \leq H\left(F\left(t, T\left(\theta_{n}(t)\right) x_{n}, x_{n}^{(1)}\left(\theta_{n}(t)\right), \ldots, x_{n}^{(k-1)}\left(\theta_{n}(t)\right)\right)\right), \\
&\left.F\left(t, T(t) z_{0}, z_{1}(t), \ldots, z_{k-1}(t)\right)\right)+\left\|f(t)-f_{n}(t)\right\| \\
& \leq\left.m(t) \max \left\{\| T\left(\theta_{n}(t)\right) x_{n}-T(t) z_{0}\right)\left\|_{\infty}, \max _{1 \leq i \leq k-1}\right\| x_{n}^{(i)}\left(\theta_{n}(t)\right)-z_{i}(t) \|\right\} \\
& \quad+\left\|f(t)-f_{n}(t)\right\| .
\end{aligned}
$$

Since the last term converges to 0 , we get $f(t) \in F\left(t, T(t) z_{0}, z_{1}(t), \ldots, z_{k-1}(t)\right)$ a.e on $[0, \tau]$. Finally, set $x(t)=z_{0}(t)$ for all $t \in[-a, \tau]$. For all $i=0, \ldots, k$ and all $t \in[0, \tau]$, we have $x^{(i)}(t)=z_{i}(t)$. Hence

$$
x^{(k)}(t)=z_{k}(t)=\dot{z}_{k-1}(t)=f(t) \in F\left(t, T(t) x, x^{(1)}(t), \ldots, x^{(k-1)}(t)\right) \text { a.e on }[0, \tau] .
$$

Also we have $x(t) \in C(t)$ and $x^{(i)}(t) \in \Omega_{i}$, on $[0, \tau]$, for all $i=1, \ldots, k-1$.

\section{REFERENCES}

[1] M., Aitalioubrahim, S., Sajid, Second-order viability result in Banach spaces, Discuss. Math. Differ. Incl. Control Optim., 30 (2010), 5-21.

[2] M., Aitalioubrahim, A viabillity result for functional differential inclusions in Banach spaces, Miskolc Mathematical Notes, Vol. 13, No. 1 (2012), 3-22.

[3] M., Aitalioubrahim, S., Sajid, Higher order viability problem in Banach spaces, Electron. J. Differential Equations, No. 30 (2012), 1-13.

[4] M., Aitalioubrahim, Viability result for second-order functional differential inclusions, J. Dyn. Control Syst., 23, No. 1 (2017), 145-153.

[5] M., Aitalioubrahim, On viability result for first order functional differential inclusions, Mat. Vesnik, 70, 4 (2018), 283-291.

[6] H., Brézis, F.E., Brouder, A general principle on ordered sets in nolinear functional analysis, Advances in Math., 21(3) (1976), 355-364.

[7] C., Castaing, M. Valadier, Convex analysis and measurable multifunctions, Lecture Notes in Mathematics 580, Springer-Verlag, Berlin-Heidelberg-New York, 1977.

[8] T. X., Duc Ha, Existence of viable solutions for nonconvex valued differential inclusions in Banach spaces, Portugal. Math., Vol. 52, Fasc. 2 (1995), 241-250.

[9] A., Gavioli, L., Malaguti, Viable solutions of differential inclusions with memory in Banach spaces, Portugal. Math., Vol. 57, Fasc. 2 (2000), 203-217.

[10] G., Haddad, Monotone trajectories of differential inclusions and functional differential inclusions with memory, Israel J. Math., 39 (1981), 83-100.

[11] G., Haddad, Monotone trajectories for functional differential inclusions, J. Differential Equations, 42 (1981), 1-24.

[12] V., Lupulescu, M., Necula, A viable result for nonconvex differential inclusions with memory, Portugal. Math., Vol. 63, Fasc. 3 (2006), 335-349.

[13] L., Marco, J. A., Murillo, Viability theorems for higher-order differential inclusions, Set-Valued Anal., 6 (1998), 21-37.

[14] Q., Zhu, On the solution set of differential inclusions in Banach spaces, J. Differential Equations, No. 93 (1991), 213-237.

University Sultan Moulay Slimane, Faculty polydisciplinary, BP 592, Mghila, Beni Mellal, Morocco.

Myelkebir Aitalioubrahim: aitalifr@hotmail.com

Received 26/02/2020 